\title{
INTERNATIONAL JOURNAL OF FLUID MECHANICS RESEARCH
}

\section{INDEX VOLUME 44, 2017}

\section{Page Range of Issues}

Issue 1: 1-92; Issue 2: 93-183; Issue 3: 185-275; Issue 4: 277-373; Issue 5: 375-468; Issue 6: 469-563

\section{ISSUE 1}

Magnetohydrodynamic Flow and Heat Transfer to Sisko Nanofluid over a Wedge M. Madhu, C.S. Reddy, \& N. Kishan

Steady MHD Mass Transfer Flow in Presence of Heat Sink and Chemical Reaction

N. Ahmed

Two-Phase Flow Field in a Cylindrical Hydrocyclone with Tangential Discharge M. Piller, J. Mencinger, G. Schena, \& G. Belardi

Modeling of Electrostatic Precipitator and Study of Particle Collection X. Zhou, X. Chen, \& H.-S. Dou

Assessment of Accumulation Rate of LDL Species in Arterial Wall Layers under Hypertension and Hyperlipidemia Conditions S. Santra, D.K. Mandal, \& S. Chakrabarti

\section{ISSUE 2}

Three-Dimensional Instability of Non-Newtonian Viscoelastic Liquid Jets Issued into a Streaming Viscous (or Inviscid) Gas M.F. El-Sayed, G.M. Moatimid, F.M.F. Elsabaa, \& M.F.E. Amer

Analysis of Transverse Vibrational Response and Instabilities of Axially Moving CNT Conveying Fluid S. Oveissi, D. Toghraie, \& S.A. Eftekhari

Elucidation of Longitudinally Grooved-Riblets Drag Reduction Performance using Pressure Drop Measurements H.A. Abdulbari, H.D. Mahammed, Z.B. Yaacob, \& W.K. Mahmood

FD Analysis of Flow Pattern Inside a Mach 3 Blowdown Supersonic Wind Tunnel on Start-Up and Steady State Operation Conditions M. Rabani, M. Dehghan Manshad, \& R. Rabani

Turbulent Wakes behind Bed-Mounted Hemispheres in Co-Existing Surface Wave K. Barman \& K. Debnath

\section{ISSUE 3}

Convection Flow and Heat Transfer in a Square Cavity with Non-Newtonian Cross Nanofluid J. Zhao, L. Zheng, \& X. Zhang

Effect of Additives on the Rheological Properties of Drilling Fluid Suspension Formulated by Bentonite with Water S.K. Dewangan \& S.L. Sinha

Vortex Flow with a Free Surface: Comparison of Analytical Solutions with Experimentally Observed Liquid Particle Trajectories A.V. Kistovich, T.O. Chaplina, \& E.V. Stepanova

Micropolar Fluid Flow through a Cylinder and a Sphere Embedded in a Porous Medium M. Krishna Prasad \& D. Srinivasacharya

Thermodiffusion and Diffusion - Thermo Effects on MHD Heat and Mass Transfer of Micropolar Fluid over a Stretching Sheet

P.S. Reddy, P. Sreedevi, \& A.J. Chamkha

Effect of Anisotropic Slip and Magnetic Field on the Flow and Heat Transfer of Eyring-Powell Fluid over an Infinite Rotating Disk N.A. Khan, A. Sohail, \& F. Sultan

ERRATUM: CFD Analysis of Flow Pattern Inside AMACH 3 Blowdown Supersonic Wind Tunnel on Start-Up and Steady State Operation Conditions 


\section{ISSUE 4}

The Measurements of Bubble Plume Structure Parameter

Influence of Nozzle Exit Conditions on the Near-Field Mean Flow Characteristics of Low-Aspect-Ratio Rectangular Jets

J. Barber, M. Saediamiri, \& M. Birouk

Evaluation of S-Shaped Diffuser Performance with Emulsion Flow (Oil in Water)

W.A. El-Askary, K.A. Ibrahim, I.M. Sakr, \& H.A. Omara

Effect of Flocculating Agents on Drag in Gravity-Driven Flow Systems

M.K.S.V. Raghav, R. Teja, \& Ch.V. Subbarao

Some Studies on Rheological and Pipeline Transportation of Concentrated Limestone-Water Slurry

S. Senapati \& J.K. Pothal

Investigation of Temperature-Dependent Viscosity on Steady, Fully Developed Mixed Convection Flow in a Vertical Microchannel: An Exact Solution

B.K. Jha \& B. Aina

\section{ISSUE 5}

An Estimation of Turbulent Secondary Flow Resistance in Rectangular Ducts from a Flow Model S.K. Bose

Effect of Synthetic Jet Parameters on Controlled Flow over an Airfoil T. Parthasarathy \& S.P. Das

The Role of an Electrically Heated Front Cover on the Thermal Performance of a Radiator F. Corvaro, M. Benucci, B. Marchetti, R.C. Grifoni, \& F. Polonara

Computationally Inexpensive Free Vortex Method to Obtain Vortex Core Position in the Wake of a Horizontal Axis Wind Turbine

I. Meghlaoui, I. Dobrev, F. Massouh, A.O. Benretem, \& D. Khalfa

Performance Optimization of Centrifugal Pump Handling Viscous Oil by Modification of Impeller Geometry

A.F. Najafi, M. Tahani, S. Govara, \& M. Najafpour

Hydrodynamic Behavior of Flow past Three Typically Arranged Circular Piers on Different Horizontal Planes

S. Das, H. Jaman, A. Chatterjee, R. Das, \& A. Mazumdar

ISSUE 6

Effect of Dimensions of Sharp Spiked Cylinder on the Buzz Phenomenon Subjected to Hypersonic Flows R. Ramachandran \& R. Sirangala Ganesh

Study of Steam-Water Direct-Contact Condensation in Steam Jet Pump A. Shah

Computational Fluid Dynamics Simulation of Disc Membrane used for Improving the Quality of Effluent Produced by the Rubber Industry

A. Banik, T.K. Bandyopadhyay, \& S.K. Biswal

Nonlinear Radiation Effect on Casson Nanofluid Past a Plate Immersed in Darcy-Brinkman Porous Medium with Binary Chemical Reaction and Activation Energy A. Zaib, M.M. Rashidi, A.J. Chamkha, \& A.F. Al-Mudhaf

Computational Modeling of Gas-Bubble Formation through a Single Submerged Orifice V.K. Prasad, S.P. Singh, \& D. Chatterjee

Influence of Geometric Parameters and Chemical Kinetics Model on Combustion in a Supersonic Flow 


\section{INTERNATIONAL JOURNAL OF FLUID MECHANICS RESEARCH Author INDEX Volume 44, 2017}

\section{Page Range of Issues}

Issue 1: 1-92; Issue 2: 93-183; Issue 3: 185-275; Issue 4: 277-373; Issue 5: 375-468; Issue 6: 469-563

Abdulbari, H.A., 131

Abdulmouti, H., 277

Ahmed, N., 15

Aina, B., 357

Al-Mudhaf, A.F., 513

Amer, M.F.E., 93

Bandyopadhyay, T.K., 499

Banik, A., 499

Barber, J., 297

Barman, K., 168

Belardi, G., 41

Benretem, A.O., 427

Benucci, M., 409

Birouk, M., 297

Biswal, S.K., 499

Bose, S.K., 375

Bykov, L.V., 553

Chakrabarti, S., 79

Chamkha, A.J., 241, 513

Chaplina, T.O., 215

Chatterjee, A., 457

Chatterjee, D., 533

Chen, X., 65

Corvaro, F., 409

Das, R., 457

Das, S., 457

Das, S.P., 387

Debnath, K., 168

Dehghan Manshad, M., 155, 275

Dewangan, S.K., 195

Dobrev, I., 427

Dou, H.-S., 65

Eftekhari, S.A., 115
El-Askary, W.A., 321

Elsabaa, F.M.F., 93

El-Sayed, M.F., 93

Govara, S., 445

Grifoni, R.C., 409

Ibrahim, K.A., 321

Jaman, H., 457

Jha, B.K., 357

Khalfa, D., 427

Khan, N.A., 257

Kishan, N., 1

Kistovich, A.V., 215

Krishna Prasad, M., 229

Madhu, M., 1

Mahammed, H.D., 131

Mahmood, W.K., 131

Mandal, D.K., 79

Marchetti, B., 409

Massouh, F., 427

Mazumdar, A., 457

Meghlaoui, I., 427

Mencinger, J., 41

Moatimid, G.M., 93

Molchanov, A.M., 553

Najafi, A.F., 445

Najafpour, M., 445

Omara, H.A., 321

Oveissi, S., 115

Parthasarathy, T., 387

Piller, M., 41

Platonov, I.M., 553

Polonara, F., 409

Pothal, J.K., 349
Prasad, V.K., 533

Rabani, M., 155, 275

Rabani, R., 155, 275

Raghav, M.K.S.V., 339

Ramachandran, R., 469

Rashidi, M.M., 513

Reddy, C.S., 1

Reddy, P.S., 241

Saediamiri, M., 297

Sakr, I.M., 321

Santra, S., 79

Schena, G., 41

Senapati, S., 349

Shah, A., 487

Singh, S.P., 533

Sinha, S.L., 195

Sirangala Ganesh, R., 469

Sohail, A., 257

Sreedevi, P., 241

Srinivasacharya, D., 229

Stepanova, E.V., 215

Subbarao, Ch.V., 339

Sultan, F., 257

Tahani, M., 445

Teja, R., 339

Toghraie, D., 115

Yaacob, Z.B., 131

Yanyshev, D.S., 553

Zaib, A., 513

Zhang, X., 185

Zhao, J., 185

Zheng, L., 185

Zhou, X., 65 


\section{INTERNATIONAL JOURNAL OF FLUID MECHANICS RESEARCH SUBJECT INDEX VOLUME 44, 2017}

Page Range of Issues

Issue 1: 1-92; Issue 2: 93-183; Issue 3: 185-275; Issue 4: 277-373; Issue 5: 375-468; Issue 6: 469-563

accumulation rate, 79

activation energy, 513

aerodynamics, 387

analytical solution, 215

anisotropic slip, 257

aspect ratio, 297

atherosclerosis, 79

axial, 115

babool gum, 195

binary reaction, 513

blade geometry, 445

Bond number, 533

Brinkman equation, 229

Brownian motion, 1

bubble dynamics, 533

bubble plume, 277

bubbly flow, 277

buoyant flow, 277

buzz, 469

carbon nanotube (CNT), 115

Casson nanofluid, 513

centrifugal pump, 445

chemical kinetics, 553

chemical reaction, 513

coalescence, 533

computational fluid dynamics,

(CFD), 41, 409, 487, 499

conditional statistics, 169

conducting, 15

convector, 409

conveying fluid, 115

coupled level set and volume of

fluid method (CLSVOF), 533

cross model, 185

cylinder, 229

cylindrical tank, 339

direct-contact condensation, 487

drag, 229

drag reduction, 131, 339

drilling fluid additives, 195

drilling fluid rheology, 195

Dufour effect, 241

DynaWhirlpool, 41

eccentric arrangement, 457

efflux time, 339

electric field, 65

electrostatic precipitator, 65

emulsion flow, 321

energy consumption, 409

energy losses, 321

equations, 553 exergy, 409

Eyring-Powell, 257

finite-element method, 241

flocculating agent, 339

flow control, 387

fluent, 499

forced convection, 185

free convection, 15

free surface flow, 277

free wake, 427

frequency, 469

front cover, 409

gambit, 499

geometric parameters, 121, 553

head loss, 349

hemisphere, 169

holdup, 321

hydrodynamics, 41, 93

hyperlipidemia, 79

hypertension, 79

incompressible, 15

inline arrangement, 457

instabilities, 115

intima, 79

isotropic beams, 375

jet-cross-flow, 387

Knudsen number, 115

limestone slurry, 349

liquid jet, 93

low solid mud, 195

Mach number, 155

magnetohydrodynamic (MHD), 241

marsh funnel, 195

membrane separation, 499

microchannel, 357

micropolar fluid, 241

mixed convection, 357

multiphase flow, 277

nanofluid, 1

natural convection, 409

Navier-Stokes equations, 553

non-equilibrium, 553

nonlocal theory, 115

non-Newtonian fluid, 79, 185

numerical analysis, 155, 445

numerical simulation, 65

open channel hydraulics, 457

oscillation, 469

particle image velocimetry (PIV), 297, 427

performance improvement, 445 pipelines, 131

porous medium, 79, 229, 513

pressure drop, 131

pulsation, 469

rectangular duct, 297, 375

Reynolds number, 297

rheology, 349

riblets, 131

root vortex, 427

rotating disk, 215, 257

rotating membrane, 499

saturation, 229

scour, 457

semi-infinite, 427

separation efficiency, 65

shear resistance, 375

shock wave, 155

side-wall, 375

simulation, 409

Sisko fluid, 1

skin friction, 131

Soret effect, 241

specific power consumption, 349

spectral analysis, 169

sphere, 229

square cavity, 185

S-shaped diffusers, 321

steam jet pump, 487

suction, 15

superhydrophobic, 257

surface flow, 277

surface trough, 215

synthetic jet, 387

temperature jump, 357

thermal analysis, 409

thermal radiation, 513

thermophoresis, 1

three-dimensional disturbances, 93

torsion, 375

transient thermal flow, 155

transient, 469

transport phenomena, 487

transverse response, 115

turbulence, 169, 457

turbulence model, 553

turbulent flow, 375

turbulent jet, 297

turn angle, 321

two-phase flow, 41, 65

unsteady, 469

velocity, 427 
velocity slip, 357

vibrational response, 115

viscoelastic fluids, 93

viscosity, 15,357

viscous fluid, 445

viscous gas, 93 volume of fluid method (VOF), 533

vortex, 215

vortex cylinder, 427

vortex dynamics, 387

vortex patch, 375

vortex ring, 427 vortex tip, 427

wave, 169

Weber number, 533

wedge, 1

wind tunnel, 155

wind turbine wake 427 